\title{
ON RESTORING FORCES WHICH ADMIT FORCING TERMS OF NON-CRITICAL AMPLITUDE
}

\author{
STEPHEN H. CRANDALL
}

Introduction. The forced vibrations of a conservative system with one degree of freedom can be represented by the equation

$$
d^{2} x / d t^{2}+g(x)=f(t) .
$$

In a mechanical system $x$ is the displacement and $t$ is the time. We call $g(x)$ a restoring force and $f(t)$ a forcing term. Throughout this paper $f(t)$ will be periodic and when we speak of a periodic solution of (1) we always mean a solution having the same period as $f(t)$.

If $g(x)$ is simply a constant multiplied by $x$, equation (1) represents a linear oscillator. In this case the amplitude of the forced oscillation is a constant multiple of the amplitude of the forcing function provided this latter is non-resonant. Here, a change in the amplitude of the forcing term merely changes the amplitude of the forced vibration. In the case of a non-linear restoring force, on the other hand, we usually expect $[1]^{1}$ that changing the amplitude of the forcing term will alter the essential form of the periodic solution.

However, as we shall show, there is a class of non-linear restoring forces, for which forcing terms exist with the property that as the amplitude of the forcing function is varied, the periodic solutions resulting all have the same form but vary only in amplitude. A general method is given for determining such a forcing function whenever it exists. As an example, the Duffing equations with $g(x)= \pm x+b x^{3}$ are discussed, and the forcing terms mentioned are found to exist and are given explicitly in (16).

Definitions. A function $f(t)$ with period $4 T$ is called a forcing term of non-critical amplitude for the system (1) if there is a solution to (1), $x(t)$, having period $4 T$ and if a factor $h(k)$ exists so that $y=h x(t)$ is a solution of

$$
d^{2} y / d t^{2}+g(y)=k f(t)
$$

for a continuous range of $k$ which includes $k=1$. In the following, we limit our discussion to odd restoring forces and for brevity will only define them for positive $x$, remembering always that $g(-x)=-g(x)$ and $g(0)=0$.

Received by the editors January 9, 1947.

${ }^{1}$ Numbers in brackets refer to the references cited at the end of the paper. 
THEOREM I. The only restoring functions, $g(x)$, out of the class of functions possessing a derivative for $x>0$, which permit forcing terms of non-critical amplitude have the form

$$
g(x)=a x+b x^{\alpha}
$$

or

$$
g(x)=a x+b x \log x
$$

where $a, b$ and $\alpha$ are constants.

Assume that in (1), $f(t)$ is in fact a restoring force of non-critical amplitude, and that $x(t)$ is a solution of period $4 T$, then $h(k) x(t)$ will be a solution of (2). Substituting for $f(t)$ in (2) its value in (1) gives

$$
d^{2} x / d t^{2}+[g(h x)-k g(x)] /(h-k)=0 .
$$

Since $x(t)$ is independent of $k$, we must have

$$
[g(h x)-k g(x)] /(h-k)=G(x),
$$

say, independent of $k$, that is, $h(k)$ must be taken so that $G$ is a function of $x$ only. The possibility of doing this is limited by the form of $g(x)$ as we shall see. Equation (5) can be rearranged to read:

$$
G(x)-g(x)=[g(h x)-h g(x)] /(h-k) .
$$

Now the term on the right of (6) is also a function of $x$ alone. Since the denominator is a function of $k$ alone, the numerator must be separable into the product of a function of $x$ with a function of $k$. Then the logarithmic derivative of the numerator with respect to $x$ will be a function of $x$ alone. Finally if we set $x=1$ in this logarithmic derivative, we obtain

$$
\frac{h g^{\prime}(h)-h g^{\prime}(1)}{g(h)-h g(1)}=\mathrm{a} \text { constant, }
$$

$\alpha$, say. Solving this differential equation we obtain

$$
g(h)=\left\{\left[g^{\prime}(1)-\alpha g(1)\right] h+\left[g(1)-g^{\prime}(1)\right] h^{\alpha}\right\} /(1-\alpha)
$$

for $\alpha \neq 1$. If $\alpha=1$, we find $g(h)=\left[g^{\prime}(1)-g(1)\right] h \log h+g(1) h$. These results are of the form stated in the theorem. It is an easy matter to verify for these forms that $h(k)$ can in fact be taken so that (5) becomes a function of $x$ alone. A practical condition, namely the requirement that the velocity $d x / d t$ be finite, limits the range of allowable values of $\alpha$ to $\alpha>-1$.

We shall now construct the non-critical forcing terms for the restor- 
ing forces of (3). We first choose $h(k)$ so that (5) is independent of $k$. There is a degree of freedom in this choice for, as we have seen, the right side of (6) must be the product of a function of $x$ with a function of $k$ and in choosing $h(k)$ what we do is constrain the function of $k$ to be a constant. The magnitude of this constant is at our disposal. Call it $C$. Then (6) becomes

$$
G(x)-g(x)=C G_{1}(x)
$$

say, and (4) becomes

$$
d^{2} x / d t^{2}+g(x)+C G_{1}(x)=0 .
$$

It is convenient to note at this point that $x(t)$, the solution of (1) when $f(t)$ is a forcing term of non-critical amplitude, is also according to (8) the free vibration solution of a conservative system with an odd restoring force. This gives us considerable information regarding $x(t)$; for example, it is completely symmetric, that is, its properties are entirely determined by its behavior during the quarter period interval, $T$, between the maximum amplitude position and the zero amplitude position. This is a not surprising consequence of our initial restriction that $g(x)$ be odd. To insure that (1) actually possesses a forcing term of non-critical amplitude with a certain given period $4 T$, we must verify that (8) has a solution $x_{0}(t)$ with period $4 T$. If $d x / d t=0$ when $x=A$, the quarter period time, $\tau$, is given by [2]

$$
\tau=\int_{0}^{A} d x /\left(\int_{x}^{A}\left[g(\xi)+C G_{1}(\xi)\right] d \xi\right)^{1 / 2} .
$$

If, by suitably choosing $A$ and $C, \tau$ can be made equal to $T$, call the corresponding solution of $(8) x_{0}(t)$. From this the required forcing term of non-critical amplitude is constructed as

$$
f(t)=d^{2} x_{0} / d t^{2}+g\left(x_{0}\right) .
$$

Note that this has the same symmetry as $x_{0}(t)$. When (10) is placed in (1), it is clear that $x_{0}(t)$ is a solution of (1). Moreover if this $f(t)$ is multiplied by $k$, we find by virtue of (4) and (8) that $h x_{0}(t)$ is indeed a solution. We have thus proved the following:

THEOREM II. A sufficient condition for (1) to possess a forcing term of non-critical amplitude with period $4 T$ when the restoring force has the form of (3) is that numbers $A$ and $C$ can be found which make $\tau$ of (9) equal to $T$.

For any given $g(x)$ of the form (3) a study of (9) will reveal the range of periods for which forcing terms of non-critical amplitude 
exist. For example, if $g(x)=a x+b x^{\alpha}$ with $a$ and $b$ positive and $-1<\alpha$, a little study shows that

$$
0<\tau<\pi / 2 a^{1 / 2} \text {. }
$$

Example. As an illustration, we find the non-critical forcing terms for the Duffing equations [1]

$$
d^{2} x / d t^{2} \pm x+b x^{3}=f(t), \quad b>0 .
$$

Equation (8) for these cases becomes

$$
d^{2} x / d t^{2} \pm x+C x^{3}=0
$$

which can be solved by quadratures. As the integrals involved are elliptic, it is somewhat easier to try an elliptic function with undetermined parameters. In this way it is found that

$$
x=\operatorname{Acn}(K t / T, k),
$$

where $k$ is the modulus and $K$ is the real quarter period of the Jacobi elliptic functions, is a solution of (12) providing

$$
\begin{aligned}
& T^{2}= \pm K^{2}\left(1-2 k^{2}\right), \\
& A^{2}=2 K^{2} k^{2} / C T^{2} .
\end{aligned}
$$

Thus non-critical forcing terms for (11) exist, with periods $4 T$, when, with the upper sign

$$
0<k<2^{1 / 2} / 2, \quad 0<T<\pi / 2
$$

and with the lower sign

$$
2^{1 / 2} / 2<k<1, \quad 0<T<\infty .
$$

The forcing terms of non-critical amplitude are found to be, from (10),

$$
f(t)=A\left[b A^{2} \pm 2 k^{2} /\left(2 k^{2}-1\right)\right] c n^{3}(K t / T, k) .
$$

For a given $T, k$ and $K(k)$ are determined from (14a). The relationship between the amplitude of the forcing term and the amplitude of the solution is clearly shown in (16) and (13).

\section{REFERENCES}

1. K. O. Friedrichs and J. J. Stoker, Forced vibrations of systems with non-linear restoring force, Quarterly of Applied Mathematics vol. 1 (1943) pp. 97-115.

2. S. Timoshenko, Vibration problems in engineering, Van Nostrand, New York, 1937, p. 120.

Massachusetts Institute of Technology 